\title{
An investigation of gene-gene interactions in dose-response studies with Bayesian nonparametrics
}

\author{
Andrew L Beam ${ }^{1 *}$, Alison A Motsinger-Reif ${ }^{2,3}$ and Jon Doyle ${ }^{4}$
}

*Correspondence:

Andrew_Beam@hms.harvard.edu

${ }^{1}$ Center for Biomedical Informatics, Boston, Massachusetts

Full list of author information is available at the end of the article

\begin{abstract}
Background: Best practice for statistical methodology in cell-based dose-response studies has yet to be established. We examine the ability of MANOVA to detect trait-associated genetic loci in the presence of gene-gene interactions. We present a novel Bayesian nonparametric method designed to detect such interactions.
\end{abstract}

Results: MANOVA and the Bayesian nonparametric approach show good ability to detect trait-associated genetic variants under various possible genetic models. It is shown through several sets of analyses that this may be due to marginal effects being present, even if the underlying genetic model does not explicitly contain them.

Conclusions: Understanding how genetic interactions affect drug response continues to be a critical goal. MANOVA and the novel Bayesian framework present a trade-off between computational complexity and model flexibility.

Keywords: Dose-response, Epistasis, Bayesian nonparametric, Neural network, Machine learning

\section{Background}

Understanding the genetic factors underlying differential drug response continues to be a primary goal in pharmecogenomics and drug development. Association studies based on the use of in vitro cell lines, such as lymphoblastoid cell lines (LCLs), are becoming an attractive alternative to traditional, human based clinical trials [1-3]. Cell lines offer increased sample sizes relative to traditional studies, resulting in higher statistical power to detect genetic variants that potentially drive drug response. However, the challenges offered by these unique data has yet to be comprehensively studied and evaluated.

Recent work has shown that considering the full set dose-responses instead of summary statistics can greatly increase the power to detect trait-associated genetic variants [3-7]. This new approach is based on a statistical method known as multivariate analysis of variance or MANOVA, which is an extension to the well known analysis of variance (ANOVA) framework. Recent studies using a MANOVA-based framework have revealed genetic loci associated with differential responses in anti-cancer agents [3,4]. However, little study to date has been done to investigate the potential effect of gene-gene interactions, or epistasis, which is thought to be a critical piece in the genetic architecture of many complex phenotypes [8-10].

C 2015 Beam et al.: licensee BioMed Central. This is an Open Access article distributed under the terms of the Creative Commons Attribution License (http://creativecommons.org/licenses/by/4.0), which permits unrestricted use, distribution, and reproduction in any medium, provided the original work is properly credited. The Creative Commons Public Domain Dedication waiver (http:// creativecommons.org/publicdomain/zero/1.0/) applies to the data made available in this article, unless otherwise stated. 
The role of epistasis in complex genetic disease continues to be debated in the literature, as well as the potential of epistasis to solve the so-call missing heritability problem [11-13]. Recent work has validated the role of epistasis in complex disease in humans [14] and drosophila [15]. To date, nearly all of the work on epistasis has focused on casecontrol studies, or single-valued QTLs (e.g. gene expression). No work has yet been done to understand how epistasis may affect the unique, multiresponse data that comes from dose-response studies.

To investigate how genetic interactions may affect the power of MANOVA we performed a simulation study across several plausible models of SNP-driven dose-response involving multiple loci. In addition to investigating the MANOVA approach, we develop a novel Bayesian nonparametric model that is capable of automatically accounting for genetic interactions in multi-response data. We have previously developed a Bayesian neural network testing framework [16] for case-control studies that is capable of identifying trait-associated loci in the presence of genetic interactions in a computationally tractable way. In this study, we extend this framework to model dose-response data that contain multiple continuous responses. Through comparisons of several possible genetic models, we hope to gain insight as to how deviation from an additive model (i.e. the presence of epistasis) may affect the statistical power of each method.

\section{Methods}

\section{Multivariate analysis of variance (MANOVA)}

First we provide a brief review of the MANOVA framework for dose-response studies. In a dose-response study, a chemical is administered to a cell culture across several concentrations and a quantitative response (e.g mRNA expression or total ATP) is measured at each concentration. Until recently, the prevailing methodology in dose-response association mapping has been to fit a parametric logistic function, known as the hill-slope model, as a function of concentration $c_{i}$ :

$$
f\left(c_{i}\right)=\operatorname{Max}-\frac{\operatorname{Max}-\operatorname{Min}}{1+\left(\frac{c_{i}}{I C_{50}}\right)^{-w}}
$$

where Max,Min are the upper and lower asymptotes, and $w$ is the hill-slope. The $I C_{50}$ parameter is the concentration at which the response is $50 \%$ of maximum and is usually given special importance because it a measure of chemical potency. The $I C_{50}$ is then treated as quantitative trait and quantitative trait locus (QTL) mapping techniques attempt to identify loci that appear to be associated with this trait. In general, this approach results in a drastic loss of power relative to potential alternatives [7,17]. Recent work has shown that multivariate analysis of variance (MANOVA) has high power across a wide variety of possible dose-response relationships, making it an attractive option for genome-wide association mapping.

Let $x_{i}=<x_{i 1}, \ldots, x_{i p}>^{T}$ be a vector containing the genotypes of all $p$ markers for individual $i$, where each $x_{i j} \in\{0,1,2\}$ contains the number of instances of the minor allele. Let $y_{i}=<y_{i 1}, \ldots, y_{i k}>$ be the vector containing the responses for subject $i$ at each of the $k$ doses, where each $y_{i k} \in \mathbb{R}$. MANOVA models the conditional expected value of $y$, given $x_{i}, E\left[y_{i k} \mid x_{i}\right]$ as a linear function:

$$
E\left[y_{i k} \mid x_{i}\right]=\beta_{0}+\beta_{k}^{T} x_{i}
$$


The least-squares estimator for $B_{p \times k}=<\beta_{1}, \ldots, \beta_{p}>$ is given by $\left(X^{T} X\right)^{-1} X^{T} Y$. where $X_{n \times p}=<x_{i}, \ldots, x_{n}>^{T}$ and $Y_{n \times k}=<y_{1}, \ldots, y_{n}>$. For the case when $n<p$ or when $X$ is not full rank, as is often the case for GWASs, smaller sub-models may be fit and analyzed. For example, if 1 million markers are genotyped for only 1,000 individuals, each marker may be fit separately along with any possible confounding covariates such as age, gender, or sub-population status.

This model has several advantages including interpretability and a well understood theoretical foundation that allows for null-hypothesis significance testing. However, the linearity assumption may be somewhat restrictive and if a trait is influenced or determined by interactions between markers, this model may only partially capture the true relationship. One approach to lessen this restriction would be to explicitly include all interactions as terms in the model, and build the linear model on this expanded set of covariates. However, this quickly becomes infeasible for even $2^{\text {nd }}$ order interactions. In a study containing one million markers, there are approximately $5 * 10^{9}$ possible $2^{\text {nd }}$ order interactions. The computational issues associated with this approach make it infeasible, as do the corresponding multiple testing issues associated with testing billions or trillions of simultaneous hypotheses.

A different approach to account for interactions would be to use a class of models that relax the linearity assumption and impose very little structural constraints on the relationship between $x_{i}$ and $y_{i k}$. Neural networks are one such approach and have a rich history of success in the machine learning and genetic epidemiology $[18,19]$. Neural nets come with several theoretical guarantees that make them appealing for modeling potentially nonlinear functions. Perhaps the most germane property is given by the universal function approximation theorem [20], which states that a sufficiently complex neural network is capable of modeling any smooth function on a compact set to an arbitrary degree of precision. This theorem ensures that a neural network is capable in principle of modeling a rich class of functions between input and output. Subsumed in the class of functions neural networks can represent is the linear model used by MANOVA. Thus, if the true model is indeed linear, the relationship learned by the neural network would automatically collapse to represent the linear mapping between input and output, while if the true relationship is more complex, it will include any nonlinearities without having to specify them a priori. In the next section we present and develop a Bayesian neural network framework for dose-response studies.

\section{Bayesian neural networks}

Bayesian neural networks represent an extension to the familiar neural network framework. Recent work in [16] has shown they can successfully model genetic interactions in case-control studies. Here we present an augmented design capable of modeling multi-response data, such as the kind observed in dose-response studies.

Neural networks construct a hierarchical representation where inputs are transformed into (potentially) nonlinear features that can undergo further nonlinear transformations. For our purposes we consider networks with only one such transformation. Networks with this architecture are often described as having one hidden layer, because the graphical model describing the network has one such transformation occurring at the same hierarchical level between input and output. Figure 1 shows a graphical depiction of a neural network model for dose-response data. 


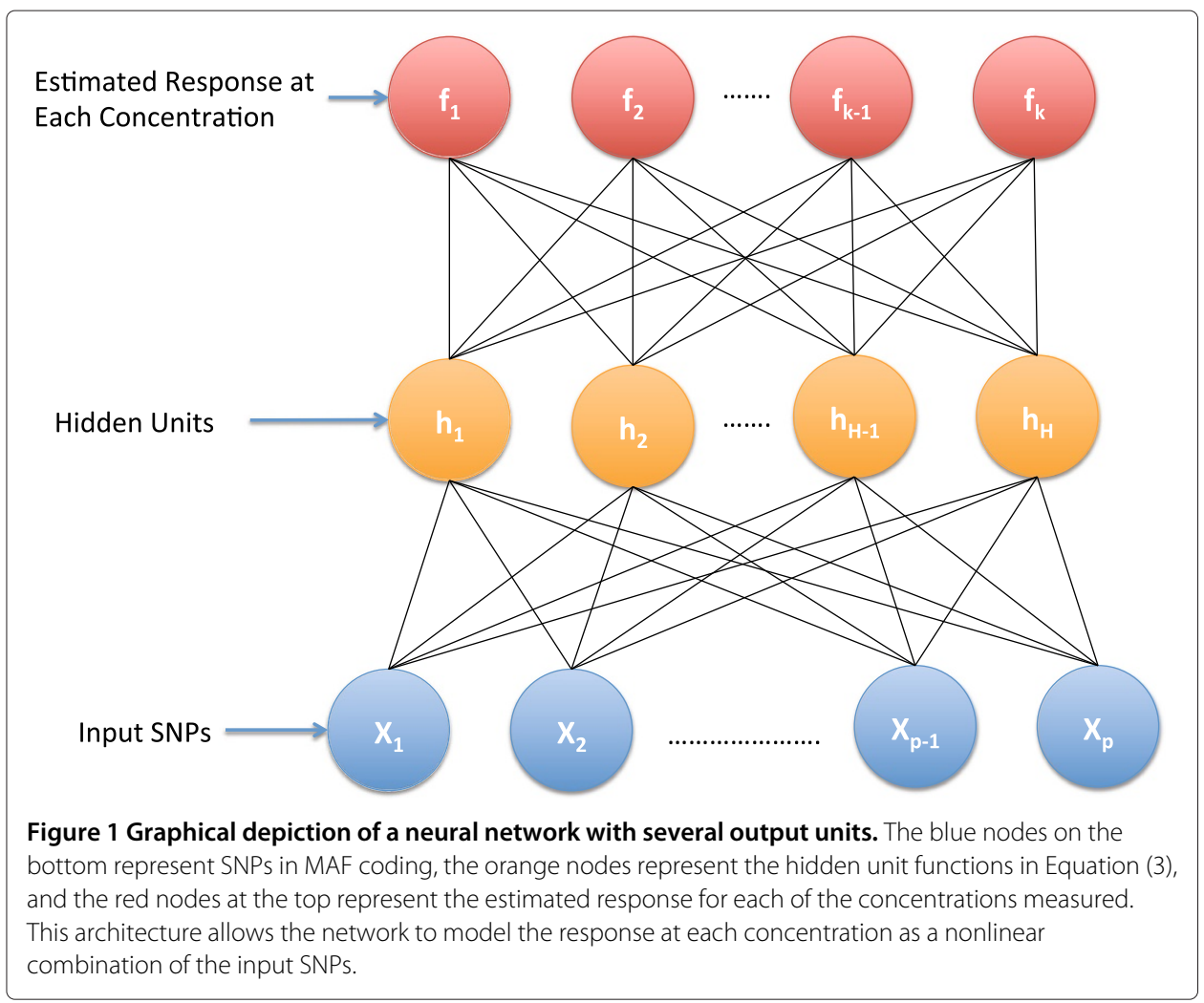

The first layer of Figure 1 shows the input SNPs represented as the number of minor alleles present at each of the $p$ markers. The inputs are fed into several hidden units, where each hidden unit, $h_{j}\left(x_{i}\right)$, performs the following nonlinear logistic transformation:

$$
h_{j}\left(x_{i}\right)=\frac{1}{1+\exp \left(-\left(a_{j}+x_{i}^{T} w_{j}\right)\right)}
$$

where $w_{j}$ is a $p \times 1$ vector of weights associated with hidden unit $j$, and $a_{j}$ is the unit's bias. The outputs from the hidden units are then fed into each output unit $\left(f_{k}\left(x_{i}\right)\right)$. Each output unit takes a linear combination of the outputs from the hidden units, using the following form:

$$
f_{k}\left(x_{i}\right)=\alpha_{k}+\sum_{j=1}^{H} h_{j}\left(x_{i}\right) * \beta_{k j}
$$

where $\alpha_{k}$ is the bias and $\beta_{k j}$ is the weight given to the output from hidden unit $j$, for output unit $k$. Equations (3) and (4) form the base neural network model. To incorporate this into a Bayesian framework, we must define several additional components. Bayesian methods define a posterior distribution over possible parameter values. The posterior distribution combines a prior distribution, that is updated based on the observed data. Letting $\theta$ represent all of the network parameters in Equations (3), (4), Bayes' rule defines the posterior distribution of $\theta$, given the data $x_{i}, y_{i}$ :

$$
p\left(\theta \mid x_{i}, y_{i}\right)=\frac{L\left(\theta \mid x_{i}, y_{i}\right) p(\theta)}{\int L\left(\theta \mid x_{i}, y_{i}\right) p(\theta) d \theta}
$$


where $p(\theta)$ is the prior distribution for the network parameters and $L\left(\theta \mid x_{i}, y_{i}\right)$ is the likelihood function. The network's output for each observation, $f_{i k}\left(x_{i}\right)$, is given an independent Gaussian likelihood with mean $f_{i k}\left(x_{i}\right)$ and unit variance, shown below:

$$
L\left(\theta \mid x_{i}, y_{i}\right)=\exp \left(-\frac{\left(y_{i k}-f_{i k}\left(x_{i}\right)\right)^{2}}{2}\right)
$$

There are many choices for the prior, $p(\theta)$, but the one adopted here is the Automatic Relevance Determination (ARD) prior [21,22]. Briefly, the ARD prior groups weights in the hidden layer together in a meaningful way. Weights that are connected to the same SNP across hidden units are given a shared parameter that controls how large the values they take on are allowed to become. Specifically, the weights in each hidden unit that are associated with SNP $j$ are given the following hierarchical prior:

$$
\begin{aligned}
& \beta_{k j} \sim N\left(0, \sigma_{j}^{2}\right) \\
& \sigma_{j}^{2} \sim I G\left(\alpha_{0}, \beta_{0}\right)
\end{aligned}
$$

where $N(\cdot, \cdot)$ and $I G(\cdot, \cdot)$ represent Normal and Inverse-Gamma densities, respectively. This prior allows the network to automatically determine which inputs are the most relevant. If SNP $j$ seems to be related to the response, then $\sigma_{j}^{2}$ will be concentrated around large values in the posterior. We have previously developed a Bayesian framework that allows for testing of variable importance relative to a null model [16]. This framework provides a posterior probability of each SNP's involvement in the response and is capable of being applied to continuous, dose-response data in addition to the case-control scenario for which it was originally developed.

The denominator of (5) is an integral that, in high-dimensions, is intractable. Thus stochastic integration techniques, such as Markov Chain Monte Carlo (MCMC) are often used. MCMC algorithms are able to draw samples from the posterior distribution $p\left(\theta \mid x_{i}, y_{i}\right)$ and only require the ability to evaluate $L\left(\theta \mid x_{i}, y_{i}\right) p(\theta)$ for specific values of $\theta$. However, popular algorithms such as the well known random-walk MetropolisHastings (RW-MH) [23,24] explore the posterior distribution too slowly to be useful in a high-dimensional setting. Recently an algorithm known as Hamiltonian Monte Carlo (HMC) has been shown to explore high-dimensional posterior distributions much more efficiently by leveraging information about the posterior's gradient, which guides the simulation to regions of high probability. This efficiency does not come free, as evaluation of the gradient must be done 10s to 1000s of times per iteration, possibility limiting HMC's usefulness when the number of parameters is large. To enable the use of HMC on complex models in high-dimensions, we have developed a GPU-based sampling scheme that allows neural network models to be used on large datasets [25]. A graphical overview of the entire Bayesian neural network procedure is provided in Figure 2.

This framework comes with several advantages relative to the MANOVA approach. As discussed previously, the relaxation of the linear assumption allows for a broader class of possible genetic architectures to be detected. Additionally, the BNN approach provides a nonparameteric procedure for variable selection while MANOVA relies on normality of errors for its testing procedure to be valid. For instance, p-values from a MANOVA analysis may not be valid for scenarios in which the number of individuals for one genotype is rare (e.g. very few homozygotes for the minor allele). Additionally, normalization procedures must often be performed in order to ensure the error distributions conform as 


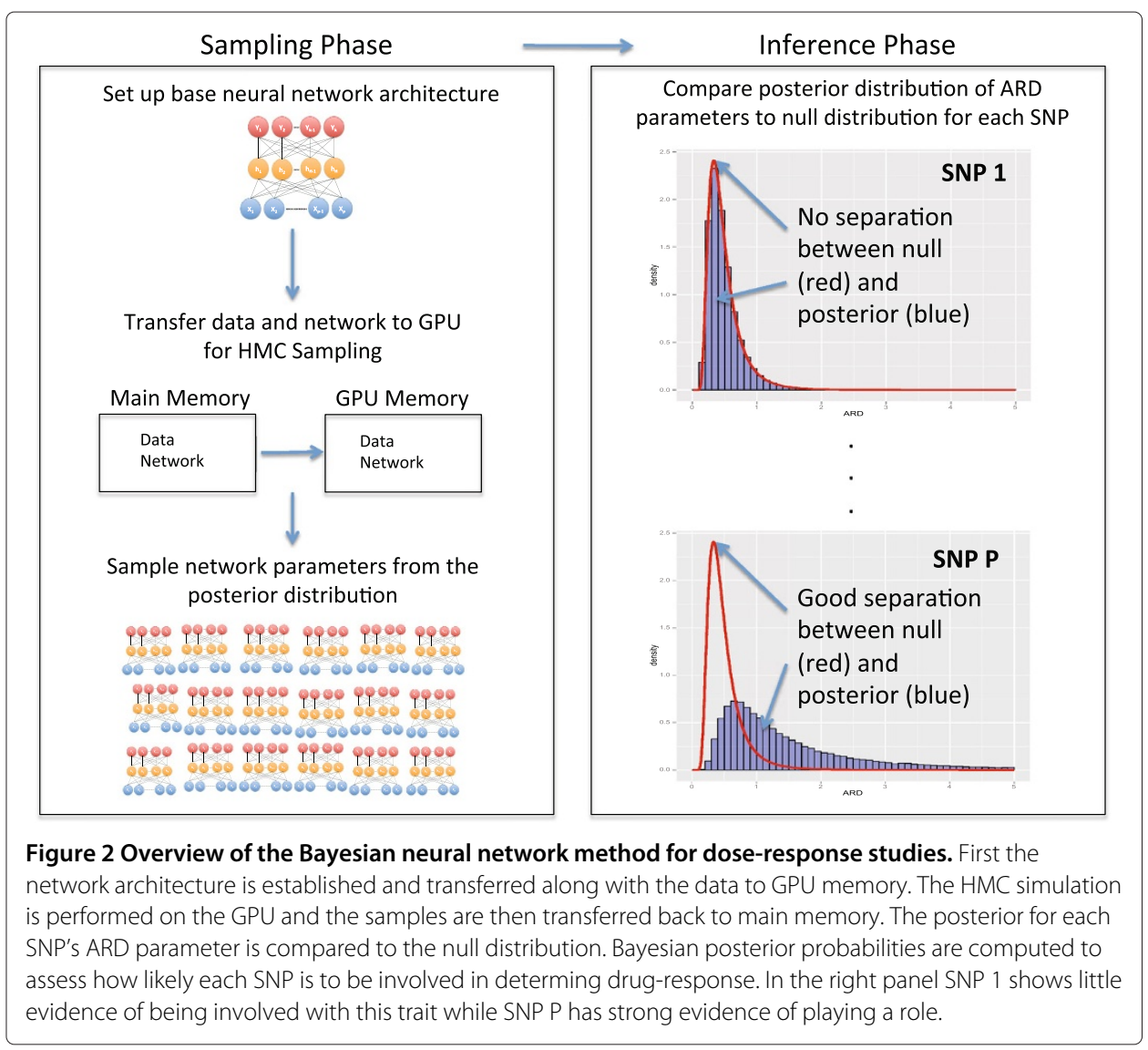

closely as possible to the assumed distribution. In contrast, due the lack of strict assumptions required by the Bayesian neural network framework, there is no reason in principle why a BNN would be affected by any of these issues.

\section{Results and discussion}

We investigated three plausible relationships between genotype and drug-response. For all simulations a Bayesian neural network with 10 hidden units was used. The HMC simulation was performed for 375 iterations, with the first 25 discarded as burn-in. For the HMC sampler a step-size value of 0.02 , a momentum-persistence value of 0.75 , and a temperature value of 1000 were used. The ARD hyper-parameter values were set to $\alpha_{0}=3$ and $\beta_{0}=1$ while the output layer used $\alpha_{0}=0.1$ and $\beta_{0}=0.1$, and we used an ARD cut-off value of 0.4. The ARD hyper-parameters, $\alpha_{0}, \beta_{0}$ control the shape and scale (respectively) of the inverse-gamma prior distribution in the ARD hierarchy. This prior distribution controls the degree to which weights in the hidden layer are constrained, relative to a standard neural network. In our experience, the network is relatively insensitive to these values but please see [16] for the explicit role these parameters play. Each response was normalized to have mean 0, unit variance. All BNN code was written in Python and is avilable at https://github.com/beamandrew/BNN.

We used a Bonferonni cut-off value of $p<0.05$ for the MANOVA procedure. MANOVA was conducted using the manova () function in R. Processing each file file 
took the BNN approximately 1 minute, while the MANOVA procedure took approximately 5 seconds for all simulation settings.

\section{Additive model}

As a baseline for the Bayesian neural network model, we first performed a simulation study using a simple additive model involving 2 loci. We generated a mean dose-response for each concentration, $\mu=<\mu_{1}, \ldots, \mu_{6}>$, according to the hill-slope model in Equation (1):

$$
\mu_{k}=1-\frac{1}{1+x_{k}^{-1.5}}
$$

for $k \in\{1, \ldots 6\}$ and $x=10^{-4} *\{0.03125,0.0625,0.10,0.25,0.5,1.25\}$, resulting in a mean dose-response curve for 6 concentrations. Deviations from the mean induced by SNP status were generated according to a linear model plus a heteroskedastic noise term, yielding an observation $i$ at each concentration, $y_{i k}$ :

$$
y_{i k}=\mu_{k} *\left(1+\frac{\theta}{2} * S_{1}-\frac{\theta}{2} * S_{2}+\epsilon\right)
$$

where $\epsilon \sim N(0,0.1)$ and $S_{1}, S_{2} \in\{0,1,2\}$ represent number of minor alleles at the two causal loci, and $\theta \in[0,1]$ represents the effect size of each SNP. In this model being homozygous for the minor allele causes a $\theta \%$ change relative to the baseline mean, $\mu_{k}$. For example, $\theta=0.02$ would correspond to a $2 \%$ change for a subject that is homozygous for the minor allele at $S_{1}$ while being homozygous for the major allele at $S_{2}$ :

$$
\begin{aligned}
& y_{k}=\mu_{k} *\left(1+\frac{0.02}{2} * 2-\frac{0.02}{2} * 0+\epsilon\right) \\
& =\mu_{k}+\mu_{k} * 0.02+\epsilon_{k}
\end{aligned}
$$

Also note that one locus $\left(S_{1}\right)$ is associated with increased drug response while the other locus $\left(S_{2}\right)$ confers a decreased average response. To evaluate the sensitivity of both MANOVA and BNN to effect size $(\theta)$ and minor allele frequency (MAF), we simulated data sets for $\theta=\{0.01 .0 .02,0.03,0.04,0.05\}$ and MAF $=\{0.01,0.05,0.1,0.2,0.3,0.4,0.5\}$. For each combination of $\theta$ and MAF, we generated 100 data sets resulting in 3500 data sets used in evaluation. Each dataset contained 2,000 observations and 998 background SNPs. Background SNPs were generated according to a random MAF, ranging uniformly from 0.01 to 0.5 . For each parameter combination, the number of times out of 100 each method correctly identified both causal SNPs as an estimate of statistical power. The results are shown in Figure 3.

Both BNNs and MANOVA display good power across a variety of parameter combinations, with MANOVA having a slight edge for a few parameter combinations. This is expected as MANOVA is testing the linear hypothesis directly, while the BNN is testing a much more general hypothesis.

\section{Additive model with interactions}

Next, we considered an additive model with an interaction term, shown below.

$$
y_{k}=\mu_{k} *\left(1+\frac{\theta}{2} * S_{1}-\frac{\theta}{2} * S_{2}+\frac{\theta}{2} * S_{1} * S_{2}+\epsilon\right)
$$




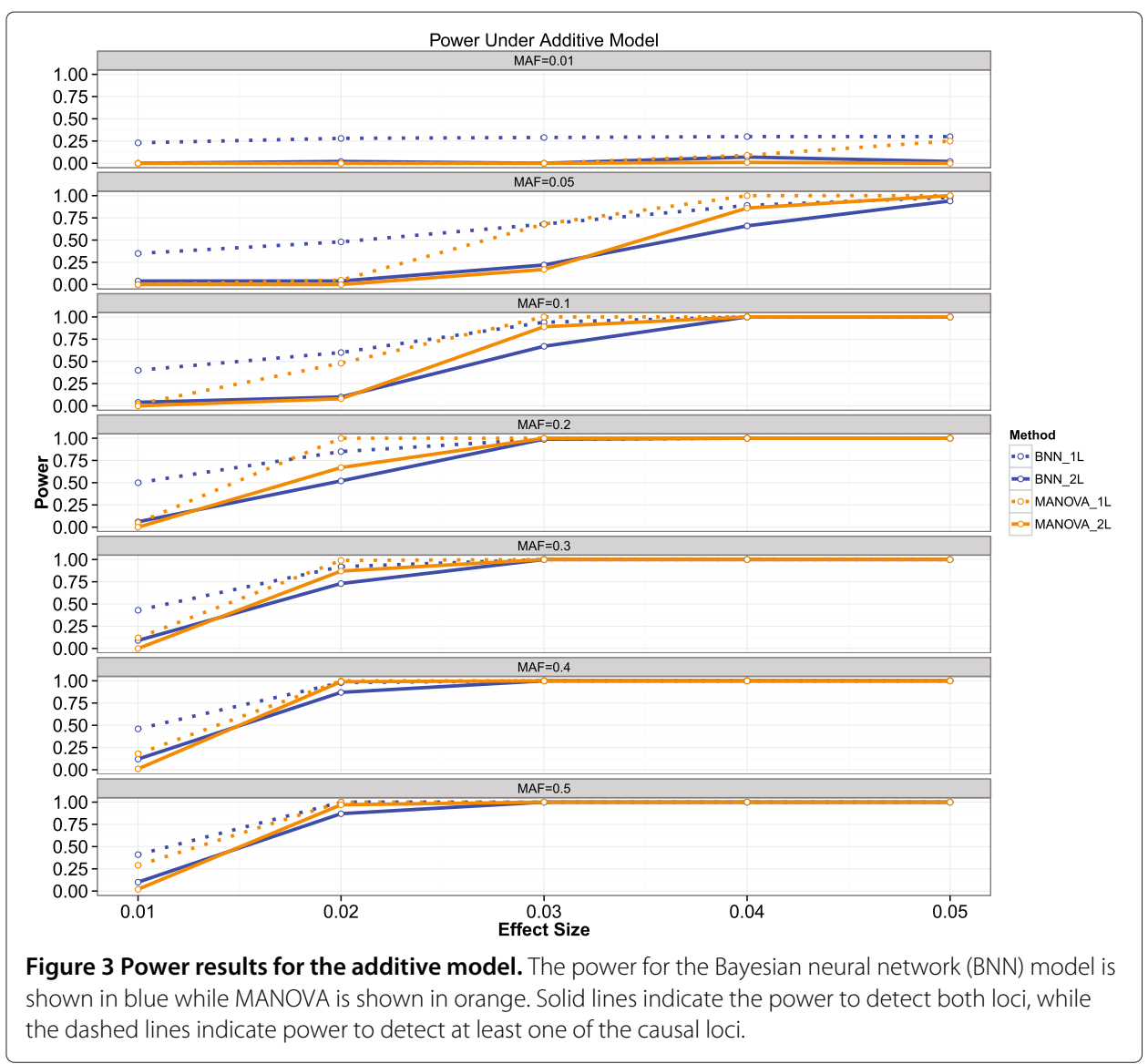

In this model there is deviation from additivity induced by the the interaction term $S_{1} * S_{2}$. We again swept over the same range of values for $\theta$ and MAF as done previously. The results are shown in Figure 4.

Again, both methods appear to have good power across a wide spectrum of parameter values. However, MANOVA does not perform as well as for larger values of MAF. The BNN also experiences a loss of power for the highest level of MAF. For most parameter combinations, the BNN outperformed MANOVA.

\section{Purely interactive model}

Finally, we investigated a model in which the SNPs affect the response only through an interaction, shown below. The results are shown in Figure 5.

$$
y_{k}=\mu_{k} *\left(1+\frac{\theta}{2} * S_{1} * S_{2}+\epsilon\right)
$$

Perhaps surprisingly, MANOVA displays good power to detect the causal loci for several parameter combinations, despite the true model ostensibly lacking any marginal effects. Both methods struggled for smaller values of MAF, but this is expected due the relatively rare nature of positive responses for small levels of MAF. 


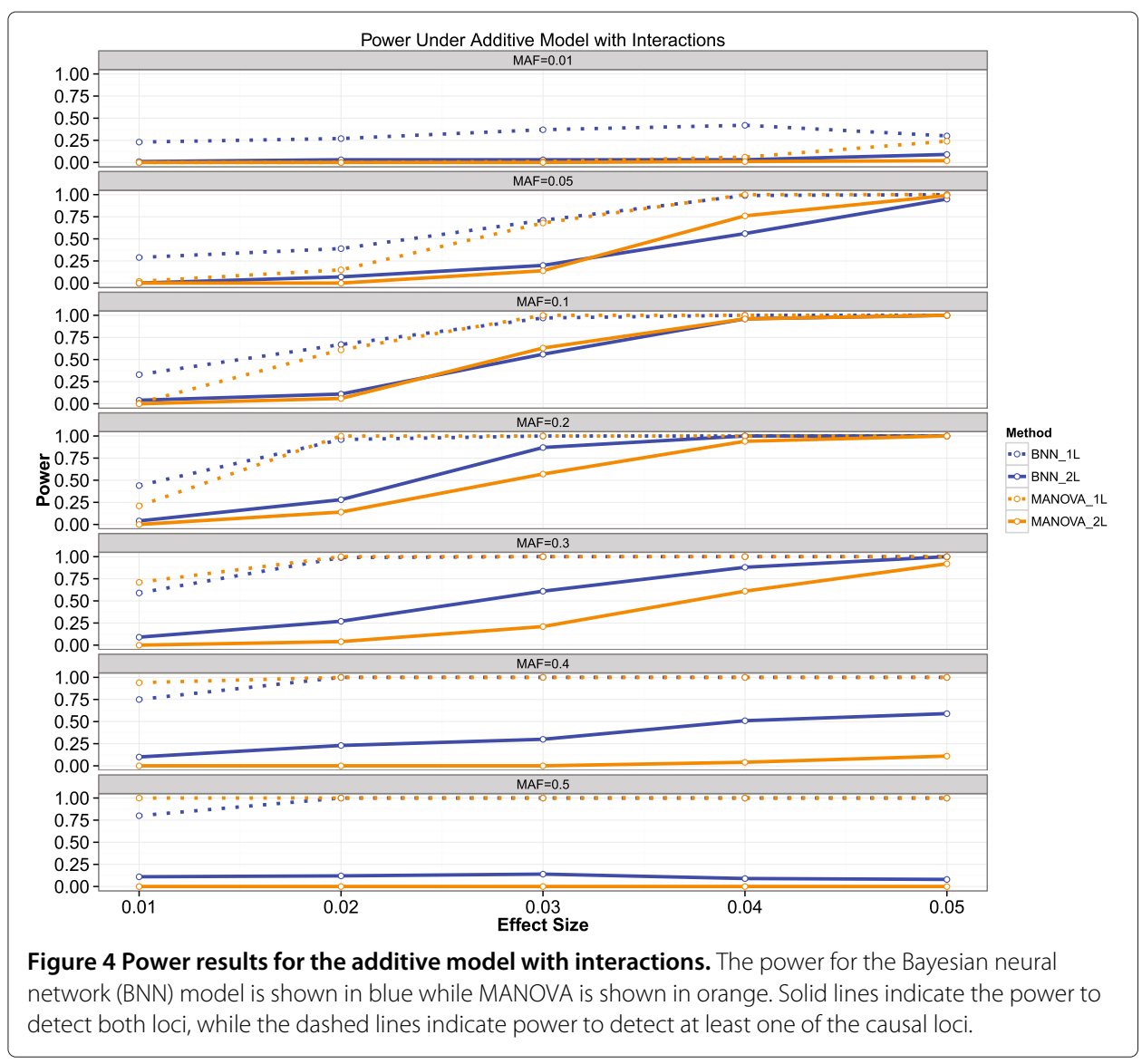

Further analysis of simulated models

The results of the preceding section motivated a more in-depth analysis of the reasons behind MANOVA's apparent ability to detect models that only contain interactions and the reduced ability by both methods to detect the model containing main effects and an interaction term. The apparent loss of power by both methods for higher values of MAF in Figure 4 may be an artifact of the specific simulation parameters used. Notice for example, that both MANOVA and BNNs have good power to detect one locus for all simulated scenarios, as evidenced by the dotted line. This suggests that the loss of power under this model is due to the decreased ability to detect the second locus $\left(S_{2}\right)$, as examination of the raw simulation results indicates this locus was rarely identified as significant. To examine this hypothesis, we will assume a more general population genetics framework and then reexamine the results of the simulated models in the previous section as specific instances of this broader viewpoint.

A marginal effect is the expected value of $y_{i k}$ given the status at one locus, averaged over all possible values for all other loci. Since the MANOVA procedure only detects marginal effects, a deeper understanding of how they may manifest in these putative models of genetic influence may be valuable. What we intend to show is that the discrete nature of the minor allele coding can introduce artifacts such as main effects in models where none are explicitly present and how marginal effects are attenuated by an interaction term in models containing both. First, we will examine the marginal effect of having a specified genotype at one locus (e.g. $S_{2}=1$ ) independently of the status at the other locus, and 


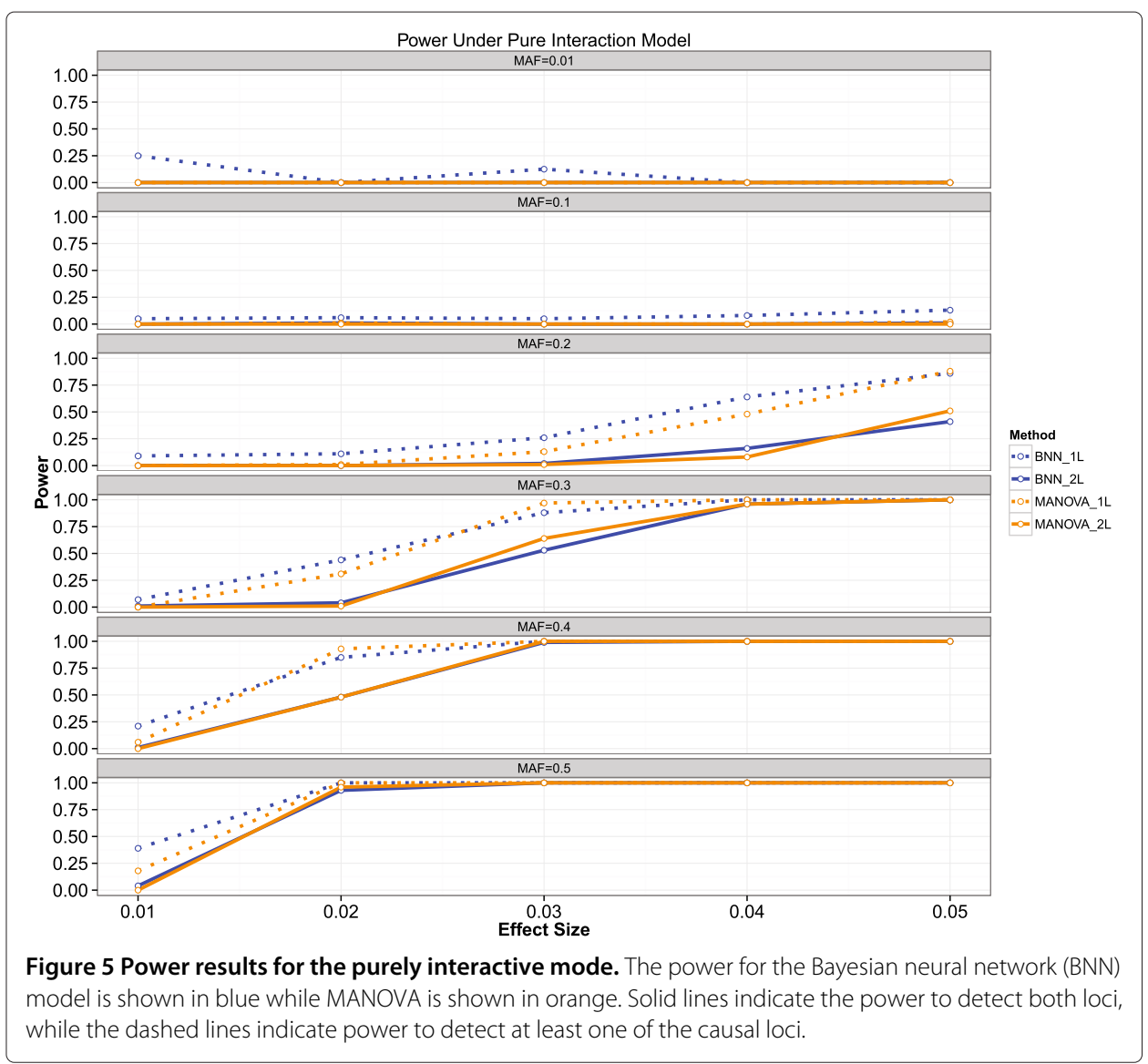

determine generally how this marginal effect changes under different simulated models as a function of effect size and MAF.

For the moment assume we an infinite population of individuals and we are interested in the relationship between two loci $S_{1}$ and $S_{2}$ and a quantitative trait, $y$. Let $p_{x y}$ be the proportion of individuals containing $x$ copies of the minor allele for locus $S_{1}$ and $y$ copies of the minor allele for locus $S_{2}$. As done previously in Equation (11), assume that the expected value of $y, E[y]$ is given by an additive relationship:

$$
E[y]=\beta_{1} * S_{1}+\beta_{2} * S_{2}
$$

where $\beta_{1}, \beta_{2}$ are the effects of having the $S_{1}, S_{2}$ genotypes, respectively. Consider the marginal effect of having the genotype $S_{2}=1$. The conditional expectation of y given $S_{2}=1, E\left(y \mid S_{2}=1\right)$, can be computed using the law of total probability, with a weighted sum over all possible values for $S_{1}$ :

$$
E\left[y \mid S_{2}=1\right]=\sum_{i=0}^{2} E\left(y \mid S_{2}=1, S_{1}=i\right) * p_{i 1}
$$

Substituting Equation (13) into (14) and simplifying:

$$
\begin{aligned}
E\left[y \mid S_{2}=1\right]= & E\left[y \mid S_{2}=1, S_{1}=0\right] \cdot p_{01}+E\left[y \mid S_{2}=1, S_{1}=1\right] \cdot p_{11} \\
& +E\left[y \mid S_{2}=1, S_{2}=2\right] \cdot p_{21} \\
= & \beta_{2} \cdot p_{01}+\left(\beta_{1}+\beta_{2}\right) \cdot p_{11}+\left(2 \beta_{1}+\beta_{2}\right) \cdot p_{21}
\end{aligned}
$$


Rearranging and simplifying the last line yields:

$$
E\left[y \mid S_{2}=1\right]=\beta_{1} \cdot\left(p_{11}+2 p_{21}\right)+\beta_{2} \cdot\left(p_{01}+p_{11}+p_{21}\right)
$$

Let the conditional expectation shown in Equation 15 be referred to as the marginal effect of $S_{2}=1$ under the additive model, or $\mu_{A}$. Now consider that instead of following a simple additive model, the expected value of $y$ is given by an additive model plus an interaction term, shown below:

$$
E[y]=\beta_{1} * S_{1}+\beta_{2} * S_{2}+\beta_{3} *\left(S_{1} \cdot S_{2}\right)
$$

Again, using a similar approach as before and summing over all possibilities for $S_{1}$ the conditional expected value is:

$$
E\left[y \mid S_{2}=1\right]=\beta_{1}\left(p_{11}+p_{21}\right)+\beta_{2}\left(p_{01}+p_{11}+p_{21}\right)+\beta_{3}\left(p_{11}+2 p_{21}\right)
$$

Let this expected value be referred to as $\mu_{A I}$. Equations (15),(17) give the marginal effect of having the genotype $S_{1}=1$ under an additive model and an additive model with an interaction for arbitrary effect sizes and for arbitrary genotype frequencies. Note that if $\beta_{1}, \beta_{2}, \beta_{3}>0$, then $\mu_{A I}>\mu_{A}$ or the marginal effect under the model with interactions would be larger, thus MANOVA would have higher power to detect this SNP as causal. However, using the values used in the simulation $\left(\beta_{1}=\beta_{3}=\frac{\theta}{2}, \beta_{2}=-\frac{\theta}{2}\right)$ the equations reduce to the following expressions:

$$
\begin{aligned}
\mu_{A} & =-\frac{\theta}{2}\left(p_{01}-p_{21}\right) \\
\mu_{A I} & =-\frac{\theta}{2}\left(p_{01}-p_{11}-2 p_{21}\right)
\end{aligned}
$$

Here it is clear that $\left|\mu_{A I}\right|<\left|\mu_{A}\right|$ since $p_{x y}>0 \forall x, y$. This is due to the negative $S_{2}$ effect, which induces a smaller marginal effect for this locus under the additive model with an interaction. This explains why in the simulations the power to detect this locus was reduced when compared to the purely additive model. The reason MANOVA has reduced power when MAF is high because the interaction term appears more often and since it has the opposite sign as the $S_{2}$ locus, it effectively cancels out the response, making it appear as if $S_{2}$ has a smaller marginal effect. Table 1 shows the values for $\mu_{A I}$ and $\mu_{A}$ for each possible level of $S_{2}$ for the effect sizes used in the simulations (i.e. $\beta_{1}=\beta_{3}=$ $\left.\frac{\theta}{2}, \beta_{2}=-\frac{\theta}{2}\right)$ :

Note that all of the marginal effects for $S_{2}$ are smaller than or equal to the additive models marginal effect size. This explains why there was a decrease in power to detect $S_{2}$ by MANOVA in the simulations.

Now we turn to the model without any explicit marginal effects and examine if there are indeed any marginal effects present. Recall the purely interactive model is $E[y]=S_{1} *$ $S_{2} * \beta_{3}$. Using the same analysis procedure as before we can calculate the marginal effect under an interactive model, $\mu_{I}$ for each level of $S_{2}$. This is shown in Table 2:

Table 1 Relationship beteween $\mu_{A I}$ and $\mu_{A}$ for each possible level of $S_{2}$

\begin{tabular}{llll}
\hline $\boldsymbol{S}_{\mathbf{2}}$ Status & $\boldsymbol{\mu}_{\boldsymbol{A} \boldsymbol{I}}$ & $\boldsymbol{\mu}_{\boldsymbol{A}}$ & $\left|\boldsymbol{\mu}_{\boldsymbol{A} \boldsymbol{I}}\right| \boldsymbol{?}\left|\boldsymbol{\mu}_{\boldsymbol{A}}\right|$ \\
\hline 0 & $\frac{\theta}{2}\left(p_{10}+2 p_{20}\right)$ & $\frac{\theta}{2}\left(p_{10}+2 p_{20}\right)$ & $=$ \\
\hline 1 & $-\frac{\theta}{2}\left(p_{01}-p_{11}-2 p_{21}\right)$ & $-\frac{\theta}{2}\left(p_{01}-p_{21}\right)$ & $<$ \\
\hline 2 & $-\frac{\theta}{2}\left(2 p_{02}-p_{12}+2 p_{22}\right)$ & $-\frac{\theta}{2}\left(2 p_{02}+p_{12}\right)$ & $<$ \\
\hline
\end{tabular}


Table 2 Relationship beteween $\mu_{I}$ and $\mu_{A}$ for each possible level of $S_{2}$

\begin{tabular}{cccc}
\hline $\boldsymbol{S}_{\mathbf{2}}$ Status & $\boldsymbol{\mu}_{\boldsymbol{I}}$ & $\boldsymbol{\mu}_{\boldsymbol{A}}$ & $\left|\boldsymbol{\mu}_{\boldsymbol{I}}\right| \boldsymbol{?}\left|\boldsymbol{\mu}_{\boldsymbol{A}}\right|$ \\
\hline 0 & 0 & $\frac{\theta}{2}\left(p_{10}+2 p_{20}\right)$ & $<$ \\
\hline 1 & $\frac{\theta}{2}\left(p_{11}+2 p_{21}\right)$ & $-\frac{\theta}{2}\left(p_{01}-p_{21}\right)$ & $?$ \\
\hline 2 & $\frac{\theta}{2}\left(p_{12}+4 p_{22}\right)$ & $-\frac{\theta}{2}\left(2 p_{02}+p_{12}\right)$ & $?$ \\
\hline
\end{tabular}

$A$ ? in the third column indicates that no strict inequality can be determined.

Here there is no clear, strict inequality, but assessments can be made for specific values of $p_{01}, p_{11}, p_{21}$. Note that in general $\left|\mu_{I}\right|>0$ in all instances so there will always be some amount of marginal effect present for this model. For many values of MAF, $\left|\mu_{I}\right|$ will be a non-trivial amount relative to $\left|\mu_{A}\right|$ resulting in high power for a model can only detect additive effects, such as MANOVA. Thus the reason MANOVA in Figure 5 has good power for high values of MAF is because there actually are marginal effects present, even though they were not explicitly included in the model.

The analysis in this section is meant as a small complement to the vast literature on quantitative trait loci (QTL) and the role of epistasis in settings other than the doseresponse framework. Several comprehensive investigations have been made using model organisms such as Drosophila melanogaster $[15,26]$ for which there is considerable evidence of the role of epistasis in quantitative traits. How the results of these simple, 2 loci models might translate to larger epistatic networks in other contexts involving many more loci is yet unclear. However, the simulated models in this study suggest that epistatic interactions in a dose-response framework may manifest as marginal effects for each loci involved in the interaction, at least for some configurations of the minor allele frequency.

\section{Analysis of the anticancer agent etoposide}

In this section we apply the the Bayesian neural network to real dose-response data originally presented in [3]. We focus on the anticancer agent Etoposide which is a topoisomerase inhibitor used in the treatment of wide variety of cancers. Etoposide was chosen for analysis due to the high heritability of cytotoxicity for this compound (approximately $40 \%$ heritable) [27] obvserved in previous studies, yet estimated marginal effects have only been able to account for a small fraction of this heritability.

The cytotoxicity of Eptoposide at six concentrations was assessed via cell viability counts in lymphoblastoid cell lines (LCLs) of 520 individuals of European descent. Genotype status for each individual was determined for either 314,621 or 620,901 SNPs, using HumanHap300 bead chip or HumanQuad610 bead chip platforms [3]. Status for 2.5 million total SNPs was imputed using the approach in [28]. Quality control on the 2.5 million SNPs was performed as in [3]. SNPs failing a test for Hardy-Weinberg equilibrium at the $p=0.01$ level were removed, which resulted in approximately 30,000 SNPs being discarded. QC and initial data preparation were performed using the software package plink [29]. In the previous section it was shown that even SNPs contributing to genetic risk through an interaction term only will still most likely result in observed marginal effect. Using this rationale we first screened for marginal SNP effects using MAGWAS [17]. Following [3], we included temperature, growth rate, the first 3 principal components, and the experimental batch as covariates in the model. After screening for SNPs with statistically significant marginal effects at the $p<10^{-5}$ level, we were left with 41 SNPs for 
analysis by the Bayesian neural network. Genome-wide screening using MAGWAS took approximately an hour on an Intel i5, quad-core desktop CPU.

We fit a neural network model with 10 hidden units, a logistic activation function and six Gaussian output units (one for each concentration). Sampling was done for 25 burnin iterations, followed by 2,000 iterations to be used for inference. The sampler settings were as follows: a of step-size $4 * 10^{-2}, L=20$ leap-frog steps per iteration, and an initial annealing temperature of $T_{0}=1000$. The ARD prior parameters were set to $\alpha_{0}=5, \beta_{0}=$ 2. Please see [16] for more detail and discussion on these parameter settings. Sampling took approximately 10 minutes on a GeForce GTX 650 desktop GPU. The HMC analysis procedure was performed for 3 independent replications and trace plots for each variable were inspected to assess if parameter estimates had converged.

The top SNP from [3] (rs2076112) has a Bayesian posterior probability which ranks it as the 20th most important SNP of the 41 SNPs passing the MAGWAS filter. As was found in [3], several experimental condition variables were found to be very important by the BNN as well. The top 7 most important variables were all related to experimental and cellular growth conditions, underscoring the importance of accounting for these conditions as covariates when performing cell-based gene-mapping. The top SNP according the BNN was rs12650820 (Bayesian probability 0.20, MAGWAS p-value $7.878 * 10^{-6}$ ), a SNP located on chromosome 4 within the follistatin-like 5 (FSTL5, location 4q32.3) gene. Dysfunction of FSTL5 has been implicated as biomarker in medulloblastoma, a highly malignant primary brain cancer [30]. All of the remaining SNPs have marginal importance scores which are suggestive of involvement, but less definitive.

\section{Conclusions}

In this study we have examined how gene-gene interactions can affect the ability to detect trait-associated loci in cell-based, dose-response association studies. We have presented a novel nonparametric procedure in the form of a Bayesian neural network and compared its performance to the MANOVA framework. Using a simulation study of plausible genetic models and a population genetics based analysis, we have shown that MANOVA may be able to detect causal loci even in the presence of genetic interactions. Additionally, we have shown that the BNN is also very capable of detecting causal loci across a range of possible genetic models. Each approach comes with trade-offs - the MANOVA approach is conceptually more simple and computationally less expensive while the BNN approach is more flexible and built upon fewer assumptions. In light of the results of this study, we recommend a two-stage approach (as was used in the Etoposide analysis) as a viable analysis strategy. MAGWAS is able to screen millions of SNPs quickly for marginal effects, which will mostly likely be present even if the underlying genetic architecture is epistatic. Next, the Bayesian neural network can be employed on a smaller subset of SNPs to explore a richer model space.

It's also worth discussing some of the modelling benefits offered by the Bayesian neural network approach. Due to flexibility afforded by the BNN framework, new constraints or data types can be easily accommodated. Note that to model a new type of data, one only must be able to write down the likelihood and incorporate this into the output layer of the network. Thus, when analysing a new type of data, the only change that must be made is the type of output layer used, which reflects the new data's likelihood function. Everything else, such as input layer, hidden layers, and ARD-testing framework, remain 
the same. There is no assumed model and no distributional assumptions, making this proposed framework very widely applicable to a variety of association mapping scenarios. More work remains to be done, however, before this approach can be used in a more turnkey manner. The most immediate improvement that can be made is to the HMC-based sampler. As with any MCMC method, sub-optimal parameter values will result in a chain failing to converge. The HMC-MCMC sampler used here is no exception and requires several tuning parameters that can dramatically impact the performance of the approach as a whole. Most often, several pilot runs are performed to find good values for these parameters. Recently, several groups have improved upon the generic HMC sampling scheme we used [31-33] which alleviate some of these issues. Incorporating these recent advances in HMC sampling techniques will help to make the method more robust and user-friendly.

In this study we have investigated how genetic interactions may affect the MANOVA analysis procedure, provided a deeper understanding of how these interactions may affect dose-response data, and presented a novel Bayesian nonparametric analysis technique. Cell-based dose-response studies hold much promise for pharmacogenomics, and the work presented here will help future research efforts utilizing this type of data.

\section{Software availability}

Software implementing the approaches outlined in this paper is available at https : / / github. com/beamandrew/BNN.

Competing interests

The authors declare that they have no competing interests.

\section{Authors' contributions}

ALB conceived of and implemented the method, and contributed to the manuscripts text. AMR was invovled with the MANOVA methodology and designing the simulation experiment and edited the text. JD helped to conceive of the methodology and contributed to the manuscript's text. All authors read and approved the final manuscript.

\section{Acknowledgements}

AMR received funding from 1R01CA161608 from the National Cancer Institute. ALB received funding form NIEHS Bioinformatics Training Grant 5T32ES007329-14 - Ruth L. Kirschstein National Research Service Award (NRSA) Institutional Research Training Grants (T32).

\section{Author details}

${ }^{1}$ Center for Biomedical Informatics, Boston, Massachusetts. ${ }^{2}$ Bioinformatics Research Center, North Carolina State University, Raleigh, North Carolina. ${ }^{3}$ Department of Statistics, North Carolina State University, Raleigh, North Carolina.

${ }^{4}$ Department of Computer Science, North Carolina State University, Raleigh, North Carolina.

Received: 7 July 2014 Accepted: 18 January 2015

Published online: 06 February 2015

\section{References}

1. Welsh M, Mangravite L, Medina MW, Tantisira K, Zhang W, Huang RS, et al. Pharmacogenomic discovery using cell-based models. Pharmacol Rev. 2009;61(4):413-29.

2. Wheeler HE, Dolan ME. Lymphoblastoid cell lines in pharmacogenomic discovery and clinical translation. Pharmacogenomics. 2012;13(1):55-70.

3. Brown CC, Havener TM, Medina MW, Jack JR, Krauss RM, McLeod HL, et al. Genome-wide association and pharmacological profiling of 29 anticancer agents using lymphoblastoid cell lines. Pharmacogenomics. 2014;15(2):137-46.

4. Brown CC, Havener TM, Medina MW, Auman JT, Mangravite LM, Krauss RM, et al. A genome-wide association analysis of temozolomide response using lymphoblastoid cell lines reveals a clinically relevant association with mgmt. Pharmacogenetics Genomics. 2012;22(11):796

5. Brown C, Havener TM, Everitt L, McLeod H, Motsinger-Reif AA. A comparison of association methods for cytotoxicity mapping in pharmacogenomics. Front Genet. 2011;2:86

6. Brown CC, Havener TM, Medina MW, Krauss RM, McLeod HL, Motsinger-Reif AA, et al. Multivariate methods and software for association mapping in dose-response genome-wide association studies. BioData Min. 2012;5(1):21.

7. Beam A, Motsinger-Reif A. Beyond ic50s: Towards robust statistical methods for in vitro association studies. J Pharmacogenom Pharmacoproteomics. 2013;2(120):2153-645. 
8. Moore $\mathrm{JH}$. The ubiquitous nature of epistasis in determining susceptibility to common human diseases. Human Heredity. 2003;56(1-3):73-82.

9. Moore JH. A global view of epistasis. Nat Genet. 2005;37(1):13-4.

10. Carlborg Ö, Haley CS. Epistasis: too often neglected in complex trait studies? Nat Rev Genet. 2004;5(8):618-25.

11. Zuk O, Hechter E, Sunyaev SR, Lander ES. The mystery of missing heritability: Genetic interactions create phantom heritability. Proc Nat Acad Sci USA. 2012;109(4):1193-8.

12. Wood AR, Tuke MA, Nalls MA, Hernandez DG, Bandinelli S, Singleton AB, et al. Another explanation for apparent epistasis. Nature. 2014;514(7520):3-5.

13. Bahcall O. Global epistasis. Nat Genet. 2014;46(8):811.

14. Hemani G, Shakhbazov K, Westra H-J, Esko T, Henders AK, McRae AF, et al. Detection and replication of epistasis influencing transcription in humans. Nature. 2014

15. Huang W, Richards S, Carbone MA, Zhu D, Anholt RR, Ayroles JF, et al. Epistasis dominates the genetic architecture of drosophila quantitative traits. Proc Nat Acad Sci USA. 2012;109(39):15553-9.

16. Beam AL, Motsinger-Reif A, Doyle J. Bayesian neural networks for detecting epistasis in genetic association studies. BMC Bioinf. 2014;15(1):368.

17. Brown CC, Havener TM, Medina MW, Krauss RM, McLeod HL, Motsinger-Reif AA. Multivariate methods and software for association mapping in dose-response genome-wide association studies. BioData Min. 2012;5(1):1-15.

18. Motsinger-Reif AA, Ritchie MD. Neural networks for genetic epidemiology: past, present, and future. BioData Min. 2008;1(3):3.

19. Motsinger-Reif AA, Dudek SM, Hahn LW, Ritchie MD. Comparison of approaches for machine-learning optimization of neural networks for detecting gene-gene interactions in genetic epidemiology. Genet Epidemiol. 2008;32(4):325-40

20. Hornik K, Stinchcombe M, White H. Multilayer feedforward networks are universal approximators. Neural Networks. 1989;2(5):359-66

21. Neal RM. Assessing relevance determination methods using delve. Nato Asi Ser F Comput Syst Sci. 1998;168:97-132.

22. Wipf DP, Nagarajan SS. A new view of automatic relevance determination. In: Advances in Neural Information Processing Systems. Curran Associates, Inc.; 2007. p. 1625-32.

23. Metropolis N, Rosenbluth AW, Rosenbluth MN, Teller AH, Teller E. Equation of state calculations by fast computing machines. J Chem Phys. 1953;21:1087.

24. Hastings WK. Monte carlo sampling methods using markov chains and their applications. Biometrika. 1970;57(1):97-109.

25. Beam AL, Ghosh SK, Doyle J. Fast hamiltonian monte carlo using gpu computing. ArXiv e-prints 2014;1402:4089. Provided by the SAO/NASA Astrophysics Data System.

26. Mackay TF, Stone EA, Ayroles JF. The genetics of quantitative traits: challenges and prospects. Nat Rev Genet. 2009;10(8):565-77.

27. Peters EJ, Motsinger-Reif A, Havener TM, Everitt L, Hardison NE, Watson VG, et al. Pharmacogenomic characterization of us fda-approved cytotoxic drugs. Pharmacogenomics. 2011;12(10):1407-15.

28. Li Y, Willer C, Sanna S, Abecasis G. Genotype imputation. Annu Rev Genomics Human Genet. 2009;10:387.

29. Purcell S, Neale B, Todd-Brown K, Thomas L, Ferreira MA, Bender D, et al. Plink: a tool set for whole-genome association and population-based linkage analyses. Am J Human Genet. 2007;81(3):559-75.

30. Remke M, Hielscher T, Korshunov A, Northcott PA, Bender S, Kool M, et al. Fstl5 is a marker of poor prognosis in non-wnt/non-shh medulloblastoma. J Clin Oncol. 2011;2011:3852 - 61.

31. Girolami M, Calderhead B. Riemann manifold langevin and hamiltonian monte carlo methods. J R Stat Soc: Ser B (Stat Methodology). 2011;73(2):123-214.

32. Sohl-Dickstein J, Mudigonda M, DeWeese M. Hamiltonian monte carlo without detailed balance. In: Proceedings of the 31st International Conference on Machine Learning. 2014. The Journal of Machine Learning Research. p. 719-26.

33. Shahbaba B, Lan S, Johnson WO, Neal RM. Split hamiltonian monte carlo. Stat Comput. 2014;24(3):339-49.

\section{Submit your next manuscript to BioMed Central and take full advantage of:}

- Convenient online submission

- Thorough peer review

- No space constraints or color figure charges

- Immediate publication on acceptance

- Inclusion in PubMed, CAS, Scopus and Google Scholar

- Research which is freely available for redistribution 